\title{
MRI-targeted prostate biopsy: the next step forward!
}

Emanuel Darius Cata ${ }^{1,2}$, Iulia Andras ${ }^{1,2}$, Teodora Telecan ${ }^{3}$, Attila Tamas-Szora ${ }^{4}$, Radu-Tudor Coman ${ }^{5}$, Dan-Vasile Stanca ${ }^{1,2}$, Ioan Coman ${ }^{1,2}$, Nicolae Crisan ${ }^{1,2}$

1) Urology Department, Clinical Municipal Hospital, Cluj-Napoca, Romania

2) Urology Department, Iuliu Hatieganu University of Medicine and Pharmacy, Cluj-Napoca, Romania

3) Faculty of Medicine, Iuliu Hatieganu University of Medicine and Pharmacy, Cluj-Napoca, Romania

4) Radiology Department, Clinical Municipal Hospital, Cluj-Napoca, Romania

5) Epidemiology Department, Iuliu Hatieganu University of Medicine and Pharmacy, Cluj-Napoca, Romania

DOI: $10.15386 / \mathrm{mpr}-1784$

Manuscript received: 19.06.2020

Received in revised form: 13.09 .2020

Accepted: 21.10.2020

Address for correspondence:

dr.iuliaandras@gmail.com

This work is licensed under a Creative Commons Attribution-NonCommercialNoDerivatives 4.0 International License

\begin{abstract}
Aim. For decades, the gold standard technique for diagnosing prostate cancer was the 10 to 12 core systematic transrectal or transperineal biopsy, under ultrasound guidance. Over the past years, an increased rate of false negative results and detection of clinically insignificant prostate cancer has been noted, resulting into overdiagnosis and overtreatment. The purpose of the current study was to evaluate the changes in diagnosis and management of prostate cancer brought by MRItargeted prostate biopsy.
\end{abstract}

Methods. A critical review of literature was carried out using the Medline database through a PubMed search, 37 studies meeting the inclusion criteria: prospective studies published in the past 8 years with at least 100 patients per study, which used multiparametric magnetic resonance imaging as guidance for targeted biopsies.

Results. In-Bore MRI targeted biopsy and Fusion targeted biopsy outperform standard systematic biopsy both in terms of overall and clinically significant prostate cancer detection, and ensure a lower detection rate of insignificant prostate cancer, with fewer cores needed. In-Bore MRI targeted biopsy performs better than Fusion biopsy especially in cases of apical lesions.

Conclusion. Targeted biopsy is an emerging and developing technique which offers the needed improvements in diagnosing clinically significant prostate cancer and lowers the incidence of insignificant ones, providing a more accurate selection of the patients for active surveillance and focal therapies.

Keywords: Fusion targeted biopsy, In-Bore MRI targeted biopsy, multiparametric MRI, prostate cancer detection, systematic prostate biopsy, targeted prostate biopsy

\section{Introduction}

Prostate cancer $(\mathrm{PCa})$ is the most frequent malignancy in male patients, being held accountable for $19 \%$ of diagnosed cancers in the American male population [1], with the third-highest mortality rate (10.1 deaths/100000 men) [2].

The former gold standard technique recommended by the European Association of Urology (EAU) for diagnosing $\mathrm{PCa}$ was represented by 10 to 12 core systematic transrectal or transperineal biopsy
(SBx), under ultrasound guidance, performed whenever the digital rectal exam is positive or the PSA above the cutoff value [3]. However, basing the indication of systematic biopsies solely on these criteria has led over the past years to an increased rate of false negative results (22-30\%) [4] and detection of clinically insignificant $\mathrm{PCa}$, resulting into overdiagnosis and overtreatment [5]. Thus, a prior imaging evaluation is needed. In 2019, EAU recommended association of systematic biopsy with targeted biopsy from the suspect lesion 
identified by multiparametric MRI [6].

The advance of technology and the availability of multiparametric magnetic resonance imaging (mpMRI) in daily practice expanded its use, not only for staging purposes, but also for diagnosis and therapeutic ones, offering the possibility of targeted biopsy. Currently, three modalities are employed:

A. Cognitive targeted biopsy (Cog-TBx): the radiologist marks the suspected lesion on the MRI, while the urologist performs the visually targeted biopsy using ultrasound guidance.

B. MRI-transrectal ultrasound (TRUS) Fusion targeted biopsy (Fus-Bx): a software overlays the MRI and ultrasound image in real time, with the annotations (lesions marking) being done by the operator.

C. In-Bore MRI targeted biopsy (IB-TBx): which is performed inside de MRI gantry, by the radiologist, using magnetic field compatible biopsy kit.

The purpose of the current study was to evaluate the changes in diagnosis and management of $\mathrm{PCa}$, brought by MRI-targeted prostate biopsy.

\section{Methods}

A critical review of literature was carried out using the Medline database through a PubMed search. The searching protocol included the following terms: "prostate cancer", "transrectal biopsy", "MRI", "fusion". The inclusion criteria were: prospective trials or retrospective prospective acquired databases published during the last 8 years, use of mpMRI for guiding targeted biopsy and minimum 100 patients included per study (except for the in-bore MRI targeted biopsy, due to the limited number of studies).

\section{Results}

37 studies were selected, with characteristics detailed in table I. Thirty two of the 37 trials were single center while 5 were multicenter. Six reports included only patients with previous negative biopsies, 18 studies patients with no prior biopsy, while 11 of them included both patients with repeated or initial biopsy. Population characteristics may be observed in table II and cancer detection rates (CDR) in table III. Definition of clinically significant prostate cancer (csPCa) varies among the studies and it is detailed in table I.

Table I. Trials included in the current analysis.

\begin{tabular}{|c|c|c|c|c|c|c|c|c|c|c|c|c|}
\hline No & Study & Year & Instit. & $\begin{array}{l}\text { Pa. } \\
\text { No. }\end{array}$ & $\begin{array}{l}\text { Previous } \\
\text { Neg. } \\
\text { Biopsy }\end{array}$ & $\begin{array}{c}\text { MRI } \\
\text { Sequences }\end{array}$ & $\mathbf{T}$ & $\begin{array}{c}\text { Endorectal } \\
\text { Coil }\end{array}$ & $\begin{array}{c}\text { Evaluation } \\
\text { System }\end{array}$ & Biopsy Type & Access Type & Definition of csPCa \\
\hline 1 & $\begin{array}{l}\text { Wysock et al. } \\
\text { (PROFUS TRIAL) [7] }\end{array}$ & 2013 & Single & 125 & $\begin{array}{l}\text { No Prior/ } \\
\text { Prior neg. } \\
\text { biopsy/ } \\
\text { AS }\end{array}$ & $\begin{array}{l}\text { T1W, T2W, } \\
\text { DWI, DCE }\end{array}$ & $3 \mathrm{~T}$ & Pelvic Coil & ESUR 2012 & $\begin{array}{l}\text { FUS-TBx, COG- } \\
\text { TBx, SBx }\end{array}$ & Transrectal & $\mathrm{GS} \geq 3+4$ \\
\hline 2 & Tonttila et al. [8] & 2015 & Single & 113 & $\begin{array}{l}\text { No prior } \\
\text { biopsy }\end{array}$ & $\begin{array}{l}\text { T1W, T2W, } \\
\text { DCE, DWI, } \\
\text { ADC }\end{array}$ & $3 \mathrm{~T}$ & Pelvic Coil & $\begin{array}{l}\text { 1-4 Likehood } \\
\text { of cancer }\end{array}$ & Cog-TBx, SBx & Transrectal & $\begin{array}{c}\mathrm{GS} \geq 3+4 \text { or GS } 6 \text { with } \\
\mathrm{MCL} \geq 4 \mathrm{~mm} \text { or with }>2 \\
\text { abnormal cores }\end{array}$ \\
\hline 3 & Pepe et al. [9] & 2016 & Single & 200 & $\begin{array}{l}\text { Prior neg. } \\
\text { biopsy }\end{array}$ & $\begin{array}{l}\text { T2W, DCE, } \\
\text { DWI, Spect. }\end{array}$ & $3 \mathrm{~T}$ & Pelvic Coil & $\begin{array}{l}\text { PIRADS } \\
\text { Score }\end{array}$ & $\begin{array}{l}\text { TPM-Bx, Fus- } \\
\text { TBx, Cog-TBx }\end{array}$ & $\begin{array}{l}\text { Transperineal/ } \\
\text { Transrectal }\end{array}$ & $\mathrm{GS} \geq 6$ with $>2$ cores \\
\hline \multirow{3}{*}{4} & \multirow{3}{*}{$\begin{array}{l}\text { Delongchamps et } \\
\text { al. [10] }\end{array}$} & 2013 & Single & 127 & $\begin{array}{l}\text { No prior } \\
\text { biopsy }\end{array}$ & $\begin{array}{l}\text { T2W, ADC, } \\
\text { DCE }\end{array}$ & $1.5 \mathrm{~T}$ & $\begin{array}{l}\text { Endorectal } \\
\text { Coil }\end{array}$ & 3 point Scale & SBx, Cog-TBx & Transrectal & $\begin{array}{c}\mathrm{GS} \geq 3+4 \text { or } \mathrm{GS} 6 \text { with } \\
\mathrm{MCL} \geq 5 \mathrm{~mm}\end{array}$ \\
\hline & & 2013 & Single & 131 & $\begin{array}{l}\text { No prior } \\
\text { biopsy }\end{array}$ & $\begin{array}{l}\text { T2W, ADC, } \\
\text { DCE }\end{array}$ & $1.5 \mathrm{~T}$ & $\begin{array}{l}\text { Endorectal } \\
\text { Coil }\end{array}$ & 3 point Scale & $\begin{array}{l}\text { SBx, rigid Fus- } \\
\text { TBx }\end{array}$ & Transrectal & $\begin{array}{c}\mathrm{GS} \geq 3+4 \text { or GS } 6 \text { with } \\
\mathrm{MCL} \geq 5 \mathrm{~mm}\end{array}$ \\
\hline & & 2013 & Single & 133 & $\begin{array}{l}\text { No prior } \\
\text { biopsy }\end{array}$ & $\begin{array}{l}\text { T2W, ADC, } \\
\text { DCE }\end{array}$ & $1.5 \mathrm{~T}$ & $\begin{array}{l}\text { Endorectal } \\
\text { Coil }\end{array}$ & 3 point Scale & $\begin{array}{l}\text { SBx, elastic } \\
\text { Fus-TBx }\end{array}$ & Transrectal & $\begin{array}{c}\mathrm{GS} \geq 3+4 \text { or GS } 6 \text { with } \\
\mathrm{MCL} \geq 5 \mathrm{~mm}\end{array}$ \\
\hline \multirow{2}{*}{5} & \multirow{2}{*}{ Kam et al. [11] } & 2017 & Single & 56 & NA & $\begin{array}{l}\text { T1W, T2W, } \\
\text { DWI, DCE }\end{array}$ & $1.5 \mathrm{~T}$ & Pelvic Coil & PIRADS & Cog-TBx, SBx & Transrectal & $\mathrm{GS} \geq 3+4$ \\
\hline & & 2017 & Single & 65 & NA & $\begin{array}{l}\text { T1W, T2W, } \\
\text { DWI, DCE }\end{array}$ & $1.5 \mathrm{~T}$ & Pelvic Coil & PIRADS & FUS-TBx, SBx & Transperineal & $\mathrm{GS} \geq 3+4$ \\
\hline 6 & Kaufmann et al. [12] & 2014 & Single & 35 & $\begin{array}{l}\text { Prior } \\
\text { negative } \\
\text { biopsy }\end{array}$ & $\begin{array}{l}\mathrm{T} 2 \mathrm{~W}, \mathrm{ADC} \\
\mathrm{DCE}\end{array}$ & $1.5 \mathrm{~T}$ & $\begin{array}{l}\text { Endorectal } \\
\text { Coil }\end{array}$ & $\begin{array}{l}\text { PIRADS } \\
\text { Score }\end{array}$ & IB-TBx, SBx & Transrectal & $\begin{array}{c}\text { GS pattern } \geq 4 \text { or tertiary } \\
\text { pattern } 5, \text { PSA }>10 \mathrm{ng} / \mathrm{ml}, \\
\text { PSAD }>0.15 \mathrm{ng} / \mathrm{ml} / \mathrm{cm}^{3}\end{array}$ \\
\hline 7 & Quentin et al.[13] & 2014 & Single & 128 & $\begin{array}{l}\text { No prior } \\
\text { biopsy }\end{array}$ & $\begin{array}{c}\mathrm{T} 2 \mathrm{~W}, \mathrm{ADC} \\
\mathrm{DCE}\end{array}$ & $3 \mathrm{~T}$ & Pelvic Coil & $\begin{array}{l}\text { PIRADS } \\
\text { Score }\end{array}$ & IB-TBx, SBx & Transrectal & $\begin{array}{c}\mathrm{GS} \geq 3+4 \text { or } \mathrm{GS} 6 \text { with } \\
\mathrm{MCL} \geq 4 \mathrm{~mm}\end{array}$ \\
\hline 8 & Pokorny et al. [14] & 2014 & Single & 223 & $\begin{array}{l}\text { No prior } \\
\text { biopsy }\end{array}$ & $\begin{array}{l}\text { T2W, DCE, } \\
\text { DWI }\end{array}$ & $3 \mathrm{~T}$ & Pelvic Coil & PIRADS v1 & IB-TBx, SBx & Transrectal & $\begin{array}{c}\text { very low volume GS } 3+4 \text { / } \\
\text { low volume GS } 6\end{array}$ \\
\hline \multirow{2}{*}{9} & \multirow{2}{*}{ Arsov et al. [15] } & \multirow{2}{*}{2015} & \multirow{2}{*}{ Single } & 106 & $\begin{array}{l}\text { Prior } \\
\text { negative } \\
\text { biopsy }\end{array}$ & $\begin{array}{l}\text { T1W, T2W, } \\
\text { DWI, DCE }\end{array}$ & $3 \mathrm{~T}$ & Pelvic Coil & Likert Scale & IB-TBx & Transrectal & $\mathrm{GS} \geq 3+4$ \\
\hline & & & & 104 & $\begin{array}{l}\text { Prior } \\
\text { negative } \\
\text { biopsy }\end{array}$ & $\begin{array}{l}\text { T1W, T2W, } \\
\text { DWI, DCE }\end{array}$ & $3 \mathrm{~T}$ & Pelvic Coil & $\begin{array}{l}\text { Likert Scale, } \\
\text { PIRADS } \\
\text { Score }\end{array}$ & FUS-TBx, SBx & Transrectal & $\mathrm{GS} \geq 3+4$ \\
\hline 10 & Sonn et al.[16] & 2013 & Single & 105 & $\begin{array}{l}\text { Prior } \\
\text { negative } \\
\text { biopsy }\end{array}$ & $\begin{array}{l}\mathrm{T} 2 \mathrm{~W}, \mathrm{ADC} \\
\mathrm{DCE}\end{array}$ & $3 \mathrm{~T}$ & Pelvic Coil & $\begin{array}{c}\text { Image grade } \\
1-5\end{array}$ & SBx, Fus-TBx & Transrectal & $\begin{array}{c}\mathrm{GS} \geq 3+4 \text { or GS } 6 \text { with } \\
\mathrm{MCL} \geq 4 \mathrm{~mm}\end{array}$ \\
\hline 11 & Kuru et al. [17] & 2013 & Single & 347 & $\begin{array}{l}\text { No Prior/ } \\
\text { Prior neg. } \\
\text { biopsy }\end{array}$ & $\begin{array}{l}\text { T1W, T2W, } \\
\text { DCE, DWI, } \\
\text { ADC }\end{array}$ & $3 \mathrm{~T}$ & NA & $\begin{array}{l}\text { Low-High } \\
\text { Suspicion }\end{array}$ & $\begin{array}{l}\text { TPM-Bx, } \\
\text { Fus-TBx }\end{array}$ & Transperineal & $\mathrm{GS} \geq 3+4$ \\
\hline 12 & Rastinehad et al. [18] & 2014 & Single & 105 & $\begin{array}{l}\text { No Prior/ } \\
\text { Prior neg. } \\
\text { biopsy }\end{array}$ & $\begin{array}{l}\text { T2W, DWI, } \\
\text { ADC, DCE }\end{array}$ & $3 \mathrm{~T}$ & Pelvic Coil & $\begin{array}{l}\text { NIH Risk } \\
\text { System/ } \\
\text { Likert Scale }\end{array}$ & SBx, Fus-TBx & Transrectal & $\begin{array}{c}\mathrm{GS} \geq 3+4 \text { or GS } 6 \text { with } \\
\mathrm{MCL} \geq 50 \% \text { or with }>2 \\
\text { abnormal cores }\end{array}$ \\
\hline
\end{tabular}


Table I. Trials included in the current analysis (continuation).

\begin{tabular}{|c|c|c|c|c|c|c|c|c|c|c|c|c|}
\hline No & Study & Year & Instit. & $\begin{array}{l}\text { Pa. } \\
\text { No. }\end{array}$ & $\begin{array}{l}\text { Previous } \\
\text { Neg. } \\
\text { Bionsy }\end{array}$ & $\begin{array}{c}\text { MRI } \\
\text { Sequences }\end{array}$ & $\mathbf{T}$ & $\begin{array}{c}\text { Endorectal } \\
\text { Coil }\end{array}$ & $\begin{array}{c}\text { Evaluation } \\
\text { System }\end{array}$ & Biopsy Type & Access Type & Definition of csPCa \\
\hline \multirow[b]{2}{*}{13} & \multirow{2}{*}{ Panebianco et al. [19] } & \multirow{2}{*}{2014} & \multirow{2}{*}{ Single } & 570 & $\begin{array}{l}\text { No prior } \\
\text { biopsy }\end{array}$ & $\begin{array}{l}\text { T2W, DCE, } \\
\text { DWI }\end{array}$ & $3 \mathrm{~T}$ & $\begin{array}{l}\text { Endorectal/ } \\
\text { Pelvic Coil }\end{array}$ & \multirow{2}{*}{$\begin{array}{l}\text { PIRADS } \\
\text { Score } \\
\text { PIRADS } \\
\text { Score }\end{array}$} & SBx & Transrectal & NA \\
\hline & & & & 570 & $\begin{array}{l}\text { No prior } \\
\text { biopsy }\end{array}$ & $\begin{array}{c}\text { T2W, DCE, } \\
\text { DWI }\end{array}$ & $3 \mathrm{~T}$ & $\begin{array}{l}\text { Endorectal/ } \\
\text { Pelvic Coil }\end{array}$ & & FUS-TBx, SBx & Transrectal & NA \\
\hline 14 & Baco et al. [20] & 2015 & Single & 175 & $\begin{array}{l}\text { No prior } \\
\text { biopsy }\end{array}$ & $\begin{array}{c}\text { T2W, DWI, } \\
\text { ADC }\end{array}$ & $1.5 \mathrm{~T}$ & Pelvic Coil & $\begin{array}{l}\text { PIRADS } \\
\text { Score }\end{array}$ & FUS-TBx, SBx & Transrectal & $\begin{array}{c}\mathrm{GS} \geq 3+4 \text { or GS } 6 \text { with } \\
\mathrm{MCL} \geq 5 \mathrm{~mm}\end{array}$ \\
\hline 15 & Siddiqui et al.[21] & 2015 & Single & 1003 & $\begin{array}{l}\text { No Prior/ } \\
\text { Prior neg. } \\
\text { biopsy }\end{array}$ & $\begin{array}{l}\text { T2W, DWI, } \\
\text { DCE, Spect. }\end{array}$ & $3 \mathrm{~T}$ & $\begin{array}{l}\text { Endorectal/ } \\
\text { Pelvic Coil }\end{array}$ & $\begin{array}{l}\text { Low-High } \\
\text { Suspicion }\end{array}$ & FUS-TBx, SBx & Transrectal & $\begin{array}{c}\mathrm{GS} \geq 3+4 \text { with }>50 \% \text { of } \\
\text { core }\end{array}$ \\
\hline 16 & de Gorski et al. [22] & 2015 & Single & 232 & $\begin{array}{l}\text { No prior } \\
\text { biopsy }\end{array}$ & $\begin{array}{l}\text { T1W, T2W, } \\
\text { DCE, DWI, } \\
\text { ADC }\end{array}$ & $1.5 \mathrm{~T}$ & Pelvic Coil & Likert Scale & SBx, Fus-TBx & Transrectal & $\begin{array}{c}\mathrm{GS} \geq 3+4 \text { or GS } 6 \text { with } \\
\mathrm{MCL} \geq 4 \mathrm{~mm}\end{array}$ \\
\hline 17 & Radtke et al. [23] & 2015 & Single & 294 & $\begin{array}{l}\text { No Prior/ } \\
\text { Prior neg. } \\
\text { biopsy }\end{array}$ & $\begin{array}{l}\text { T2W, DCE, } \\
\text { DWI }\end{array}$ & $3 \mathrm{~T}$ & Pelvic Coil & $\begin{array}{l}\text { PIRADS } \\
\text { Score }\end{array}$ & $\begin{array}{l}\text { TPM-Bx, Fus- } \\
\text { TBx }\end{array}$ & Transperineal & $\mathrm{GS} \geq 3+4$ \\
\hline 18 & Cash et al. [24] & 2015 & Single & 408 & $\begin{array}{l}\text { No Prior/ } \\
\text { Prior neg. } \\
\text { biopsy }\end{array}$ & T2W, DWI & $3 \mathrm{~T}$ & $\begin{array}{c}\text { No } \\
\text { endorectal } \\
\text { coil }\end{array}$ & $\begin{array}{l}\text { PIRADS } \\
\text { Score v1 }\end{array}$ & FUS-TBx, SBx & Transrectal & $\begin{array}{c}\mathrm{GS} \geq 3+4 \text { or GS } 6 \text { with } \\
\mathrm{MCL} \geq 4 \mathrm{~mm}\end{array}$ \\
\hline 19 & Mozer et al. [25] & 2015 & Single & 152 & $\begin{array}{l}\text { No prior } \\
\text { biopsy }\end{array}$ & $\begin{array}{c}\text { T2W, DCE, } \\
\text { DWI }\end{array}$ & $1.5 \mathrm{~T}$ & Pelvic Coil & Likert Scale & FUS-TBx, SBx & Transrectal & $\begin{array}{c}\mathrm{GS} \geq 3+4 \text { or } \mathrm{GS} 6 \text { with } \\
\mathrm{MCL} \geq 4 \mathrm{~mm}\end{array}$ \\
\hline 20 & Peltier et al. [26] & 2015 & Single & 110 & $\begin{array}{l}\text { No prior } \\
\text { biopsy }\end{array}$ & $\begin{array}{l}\text { T1W, T2W, } \\
\text { DWI, DCE }\end{array}$ & $3 \mathrm{~T}$ & $\begin{array}{l}\text { Endorectal/ } \\
\text { Pelvic Coil }\end{array}$ & PIRADS & FUS-TBx, SBx & Transrectal & $\begin{array}{c}\mathrm{GS} \geq 3+4 \text { or } \mathrm{GS} 6 \text { with } \\
\mathrm{MCL} \geq 6 \mathrm{~mm}\end{array}$ \\
\hline 21 & Porpiglia et al.[27] & 2016 & Single & $\begin{array}{l}107 \\
105\end{array}$ & $\begin{array}{l}\text { No prior } \\
\text { biopsy } \\
\text { No prior } \\
\text { biopsy }\end{array}$ & $\begin{array}{l}\text { T2W, DCE, } \\
\text { DWI } \\
\text { T2W, DCE, } \\
\text { DWI }\end{array}$ & $1.5 \mathrm{~T}$ & $\begin{array}{l}\text { Endorectal } \\
\quad \text { Coil } \\
\text { Endorectal } \\
\text { Coil }\end{array}$ & $\begin{array}{l}\text { PIRADS } \\
\text { Score } \\
\text { PIRADS } \\
\text { Score }\end{array}$ & $\begin{array}{c}\text { SBx, Fus-TBx } \\
\text { SBx }\end{array}$ & $\begin{array}{l}\text { Transperineal/ } \\
\text { Transrectal } \\
\text { Transperineal/ } \\
\text { Transrectal }\end{array}$ & $\begin{array}{c}\mathrm{GS} \geq 3+4 \text { or GS } 6 \text { with } \\
\mathrm{MCL} \geq 5 \mathrm{~mm} \\
\mathrm{GS} \geq 3+4 \text { or GS } 6 \text { with } \\
\mathrm{MCL} \geq 5 \mathrm{~mm}\end{array}$ \\
\hline 22 & Zhang et al. [28] & 2016 & Single & 224 & $\begin{array}{l}\text { No prior } \\
\text { biopsy }\end{array}$ & $\begin{array}{l}\text { T2W, DCE, } \\
\text { DWI, ADC }\end{array}$ & $3 \mathrm{~T}$ & NA & PIRADS & FUS-TBx, SBx & Transperineal & $\begin{array}{c}\mathrm{GS} \geq 3+4 \text { or GS } 6 \text { with } \\
\mathrm{MCL} \geq 50 \%\end{array}$ \\
\hline 23 & Mehralivand et al. [29] & 2017 & Single & 339 & $\begin{array}{l}\text { No Prior/ } \\
\text { Prior neg. } \\
\text { biopsy }\end{array}$ & $\begin{array}{l}\text { T2W, DWI, } \\
\text { ADC, DCE }\end{array}$ & $3 \mathrm{~T}$ & $\begin{array}{l}\text { Endorectal/ } \\
\text { Pelvic Coil }\end{array}$ & $\begin{array}{l}\text { PIRADS } \\
\text { Score v2 }\end{array}$ & FUS-TBx, SBx & Transrectal & ISUP $\geq 2$ \\
\hline 24 & Hakozaki et al. [30] & 2017 & Single & 177 & $\begin{array}{l}\text { No Prior/ } \\
\text { Prior neg. } \\
\text { biopsy/ } \\
\text { AS }\end{array}$ & $\begin{array}{c}\text { T2W, DCE, } \\
\text { DWI }\end{array}$ & $3 \mathrm{~T}$ & NA & PIRADS & FUS-TBx, SBx & Transperineal & Epstein Criteria \\
\hline 25 & Gordetsky et al. [31] & 2017 & Single & 191 & NA & $\begin{array}{l}\text { T2W, DCE, } \\
\text { DWI, ADC }\end{array}$ & NA & NA & PIRADS & FUS-TBx, SBx & Transrectal & $\mathrm{GS} \geq 3+4$ \\
\hline 26 & Castellucci et al. [32] & 2017 & Single & 168 & $\begin{array}{l}\text { No prior } \\
\text { biopsy }\end{array}$ & $\begin{array}{c}\text { T2W, DCE, } \\
\text { DWI }\end{array}$ & $1.5 \mathrm{~T}$ & Surface coil & $\begin{array}{l}\text { PIRADS } \\
\text { Score v1 }\end{array}$ & Cog-TBx, SBx & Transrectal & $\mathrm{GS} \geq 3+4$ \\
\hline 27 & Mariotti et al. [33] & 2017 & Single & 100 & $\begin{array}{l}\text { No Prior/ } \\
\text { Prior neg. } \\
\text { biopsy }\end{array}$ & $\begin{array}{l}\text { T2W, DCE, } \\
\text { DWI }\end{array}$ & $3 \mathrm{~T}$ & Surface coil & Likert & FUS-TBx, SBx & Transrectal & $\mathrm{GS} \geq 3+4$ \\
\hline 28 & Boesen et al. [34] & 2017 & Single & 289 & $\begin{array}{c}\text { Prior } \\
\text { negative } \\
\text { biopsy }\end{array}$ & $\begin{array}{c}\text { T2W, DCE, } \\
\text { DWI }\end{array}$ & $3 \mathrm{~T}$ & NA & $\begin{array}{l}\text { PIRADS } \\
\text { Score v1 }\end{array}$ & FUS-TBx, SBx & Transrectal & $\begin{aligned} \mathrm{GS} & \geq 3+4 ;>50 \% \text { of core } \\
& \geq 3 \text { positive cores }\end{aligned}$ \\
\hline 29 & $\begin{array}{c}\text { Kasivisvanathan et } \\
\text { al. [35] }\end{array}$ & 2018 & Multi & 500 & $\begin{array}{l}\text { No prior } \\
\text { biopsy }\end{array}$ & $\begin{array}{l}\text { T2W, DCE, } \\
\text { DWI }\end{array}$ & $\begin{array}{c}1.5 \mathrm{~T} / \\
3 \mathrm{~T}\end{array}$ & $\begin{array}{l}\text { Endorectal } \\
\text { or Pelvic } \\
\text { Coil }\end{array}$ & PIRADS & FUS-TBx, SBx & Transrectal & $\mathrm{GS} \geq 3+4$ \\
\hline 30 & Borkowetz et al. [36] & 2018 & Multi & 214 & $\begin{array}{l}\text { No prior } \\
\text { biopsy }\end{array}$ & $\begin{array}{l}\text { T1W, T2W, } \\
\text { DWI, DCE }\end{array}$ & $3 \mathrm{~T}$ & $\begin{array}{l}\text { No endo- } \\
\text { rectal coil }\end{array}$ & $\begin{array}{l}\text { PIRADS v1 } \\
\text { and v2 }\end{array}$ & SBx, Fus-TBx & Transperineal & $\mathrm{GS} \geq 3+4$ \\
\hline 31 & Hofbauer et al. [37] & 2018 & Single & 704 & $\begin{array}{l}\text { No Prior/ } \\
\text { Prior neg. } \\
\text { biopsy }\end{array}$ & $\begin{array}{l}\text { T2W, DCE, } \\
\text { DWI }\end{array}$ & $3 \mathrm{~T}$ & Pelvic Coil & $\begin{array}{l}\text { PIRADS } \\
\text { Score v2 }\end{array}$ & FUS-TBx, SBx & Transrectal & $\mathrm{GS} \geq 3+4$ \\
\hline 32 & & 2018 & Single & 103 & $\begin{array}{l}\text { No Prior/ } \\
\text { Prior neg. } \\
\text { biopsy }\end{array}$ & $\begin{array}{c}\text { T2W, DCE, } \\
\text { DWI }\end{array}$ & $3 \mathrm{~T}$ & $\begin{array}{l}\text { Endorectal } \\
\text { Coil }\end{array}$ & $\begin{array}{l}\text { PIRADS } \\
\text { Score v2 }\end{array}$ & IB-Tx, & Transrectal & $\mathrm{GS} \geq 3+4$ \\
\hline 32 & Costa et al. [30] & 2010 & Singie & 300 & $\begin{array}{l}\text { No Prior/ } \\
\text { Prior neg. } \\
\text { biopsy }\end{array}$ & $\begin{array}{c}\text { T2W, DCE, } \\
\text { DWI }\end{array}$ & $3 \mathrm{~T}$ & $\begin{array}{l}\text { Endorectal } \\
\text { Coil }\end{array}$ & $\begin{array}{l}\text { PIRADS } \\
\text { Score v2 }\end{array}$ & FUS-TBx, SBx & Transrectal & $\mathrm{GS} \geq 3+4$ \\
\hline 33 & Maxeiner et al. [39] & 2018 & Single & 318 & $\begin{array}{l}\text { No prior } \\
\text { biopsy }\end{array}$ & $\begin{array}{c}\text { T2W, DCE, } \\
\text { DWI }\end{array}$ & $3 \mathrm{~T}$ & Surface coil & $\begin{array}{l}\text { PIRADS } \\
\text { Score }\end{array}$ & FUS-TBx, SBx & Transrectal & $\mathrm{GS} \geq 4+3$ or $\mathrm{MCCL} \geq 6 \mathrm{MM}$ \\
\hline & & & & 79 & $\begin{array}{l}\text { Prior } \\
\text { negative } \\
\text { biopsy }\end{array}$ & $\begin{array}{c}\text { T2W, DCE, } \\
\text { DWI }\end{array}$ & $3 \mathrm{~T}$ & NA & $\begin{array}{l}\text { PIRADS } \\
\text { Score v2 }\end{array}$ & FUS-TBx & Transperineal & $\mathrm{GS} \geq 3+4$ \\
\hline 34 & Wegelin et al. [40] & 2018 & Multi & 78 & $\begin{array}{l}\text { Prior } \\
\text { negative } \\
\text { biopsy }\end{array}$ & $\begin{array}{c}\text { T2W, DCE, } \\
\text { DWI }\end{array}$ & $3 \mathrm{~T}$ & NA & $\begin{array}{l}\text { PIRADS } \\
\text { Score v2 }\end{array}$ & Cog-TBx & Transrectal & $\mathrm{GS} \geq 3+4$ \\
\hline & & & & 77 & $\begin{array}{l}\text { Prior } \\
\text { negative } \\
\text { biopsy }\end{array}$ & $\begin{array}{c}\text { T2W, DCE, } \\
\text { DWI }\end{array}$ & $3 \mathrm{~T}$ & NA & $\begin{array}{l}\text { PIRADS } \\
\text { Score v2 }\end{array}$ & IB-TBx & Transrectal & $\mathrm{GS} \geq 3+4$ \\
\hline 35 & van der Leest [41] & 2019 & Multi & 626 & $\begin{array}{l}\text { No prior } \\
\text { biopsy }\end{array}$ & $\begin{array}{l}\text { T2W, DCE, } \\
\text { DWI }\end{array}$ & $3 \mathrm{~T}$ & NA & $\begin{array}{l}\text { PIRADS } \\
\text { Score v2 }\end{array}$ & IB-TBx, SBx & Transrectal & $\mathrm{GS} \geq 3+4$ \\
\hline 36 & hourvet al [42] & 2019 & & 248 & $\begin{array}{l}\text { No prior } \\
\text { biopsy }\end{array}$ & $\begin{array}{l}\text { T2W, DCE, } \\
\text { DWI }\end{array}$ & $3 \mathrm{~T}$ & Surface coil & $\begin{array}{l}\text { PIRADS } \\
\text { Score v2 }\end{array}$ & $\begin{array}{l}\text { FUS-TBx, } \\
\text { SBx }\end{array}$ & Transrectal & $\mathrm{GS} \geq 3+4$ \\
\hline 36 & houry et al. [42] & 2019 & Single & 248 & $\begin{array}{l}\text { No prior } \\
\text { biopsy }\end{array}$ & $\begin{array}{c}\text { T2W, DCE, } \\
\text { DWI }\end{array}$ & $3 \mathrm{~T}$ & Surface coil & $\begin{array}{l}\text { PIRADS } \\
\text { Score v2 }\end{array}$ & Cog-TBx, SBx & Transrectal & $\mathrm{GS} \geq 3+4$ \\
\hline 37 & Rouviere et al. [43] & 2019 & Multi & 251 & $\begin{array}{l}\text { No prior } \\
\text { biopsy }\end{array}$ & $\begin{array}{l}\text { T2W, DCE, } \\
\text { DWI }\end{array}$ & $\begin{array}{c}1.5 \mathrm{~T} / \\
3 \mathrm{~T}\end{array}$ & $\begin{array}{l}\text { Surface/ } \\
\text { endorectal } \\
\text { coil }\end{array}$ & Likert score & $\begin{array}{l}\text { Fus-TBx/Cog- } \\
\text { TBx, SBx }\end{array}$ & Transrectal & $\begin{array}{c}\mathrm{GS} \geq 3+4 \text { or GS } 6 \text { with } \geq \\
6 \mathrm{~mm}\end{array}$ \\
\hline
\end{tabular}


Table II. Population characteristics of the trials included in our analysis.

\begin{tabular}{|c|c|c|c|c|c|}
\hline No. & Study & $\begin{array}{l}\text { Number of } \\
\text { patients }\end{array}$ & Mean age & Mean PSA ng/ml & Prostate volume cc \\
\hline 1 & $\begin{array}{l}\text { Wysock et al. } \\
\text { (PROFUS TRIAL) [7] }\end{array}$ & 125 & $65(56.3-71.0)$ & $5.1(3.5-7.31)$ & $46(31.0-62.5)$ \\
\hline 2 & Tonttila et al. [8] & 113 & $62.5(56-67)$ & $6.15(4.0-10.7)$ & $29.8(23.5-44.3)$ \\
\hline 3 & Pepe et al. [9] & 200 & $61(47-75)$ & $8.6(4.5-26)$ & NA \\
\hline \multirow{3}{*}{4} & \multirow{3}{*}{ Delongchamps et al. [10] } & 127 & $62.7 \pm 7.4$ & $8.1 \pm 3.7$ & $53 \pm 25$ \\
\hline & & 131 & $64.6 \pm 6.7$ & $8.3 \pm 4.1$ & $55.7 \pm 35.1$ \\
\hline & & 133 & $64.5 \pm 7.9$ & $9 \pm 3.9$ & $58.3 \pm 28.6$ \\
\hline \multirow{2}{*}{5} & \multirow{2}{*}{ Kam et al. [11] } & 56 & $66.3(45-80)$ & $7.5(1.3-18)$ & $49.7(13-125)$ \\
\hline & & 65 & $65.0(48-75)$ & $7.3(2.7-18)$ & $55.2(16-128)$ \\
\hline 6 & Kaufmann et al. [12] & 35 & $68(55-75)$ & $9.42(4-25)$ & $42(20-66)$ \\
\hline 7 & Quentin et al. [13] & 128 & $67(62-72)$ & $6.7(5.1-9)$ & $47.5(36-66.9)$ \\
\hline 8 & Pokorny et al. [14] & 223 & $63(57-68)$ & $5.3(4.1-6.6)$ & $41(30-59)$ \\
\hline \multirow{2}{*}{9} & \multirow{2}{*}{ Arsov et al. [15] } & 106 & $66(60-71)$ & $10(7.8-14.9)$ & $54(40-77)$ \\
\hline & & 104 & $68(63-71)$ & $10.8(7.4-15.5-$ & $60(42-82)$ \\
\hline 10 & Sonn et al. [16] & 105 & $65[59-70]$ & $7.5[5-11.2]$ & $58[39-82]$ \\
\hline 11 & Kuru et al. [17] & 347 & $65.3(42-82)$ & $9.85(0.5-105)$ & $48.7(9-180)$ \\
\hline 12 & Rastinehad et al. [18] & 105 & $65.8(42-87)$ & $9.2(0.6-62.0)$ & NA \\
\hline \multirow{2}{*}{13} & \multirow{2}{*}{ Panebianco et al. [19] } & 570 & $64(51-82)$ & NA & NA \\
\hline & & 570 & $64(51-82)$ & NA & NA \\
\hline 14 & Baco et al. [20] & 175 & $65(59-69)$ & $7.3(5.5-9.9)$ & $42(30-59)$ \\
\hline 15 & Siddiqui et al. [21] & 1003 & $62.1(7.5)$ & $6.7(4.4-10.7)$ & $49(36-71)$ \\
\hline 16 & de Gorski et al. [22] & 232 & $64 \pm 6.4$ & $6.5 \pm 1.8$ & $47 \pm 24.3$ \\
\hline 17 & Radtke et al. [23] & 294 & $64(60-71)$ & $7.3 \pm 6.0$ & $47.00 \pm 37.5$ \\
\hline 18 & Cash et al. [24] & 408 & $67(60-71)$ & $9.33(0.68-14.65)$ & $50(35-65)$ \\
\hline 19 & Mozer et al. [25] & 152 & $63(59.3-67.5)$ & $6(5-7.9)$ & $44(30-55)$ \\
\hline 20 & Peltier et al. [26] & 110 & $65.1(48.0-79.2)$ & $8.4(0.7-40)$ & $49.3(18-162)$ \\
\hline \multirow{2}{*}{21} & \multirow{2}{*}{ Porpiglia et al. [27] } & 107 & $64(58-70)$ & $5.9(4.8-7.5)$ & $46.2(34.5-71.6)$ \\
\hline & & 105 & $66(60-70)$ & $6.7(5.5-8.5)$ & $45.7(34.6-65.0)$ \\
\hline 22 & Zhang et al. [28] & 224 & $69(40-85)$ & $10.05(3.61-78.39)$ & $45.5(22-77)$ \\
\hline 23 & Mehralivand et al. [29] & 339 & 64.1 & $6.47(4.59-9.31)$ & $55(41-79)$ \\
\hline 24 & Hakozaki et al. [30] & 177 & $68.3(48-89)$ & $10.9(1.65-218)$ & $42.4(11-134)$ \\
\hline 25 & Gordetsky et al. [31] & 191 & $63.3 \pm 7.2$ & $9.2 \pm 9.6$ & NA \\
\hline 26 & Castellucci et al. [32] & 168 & $61.4 \pm 7.60$ & $8.3 \pm 6.1$ & $48.9 \pm 6.7$ \\
\hline 27 & Mariotti et al. [33] & 100 & $62.5(35-86)$ & $5.3(0.2-36.0)$ & $48(16.0-130.0)$ \\
\hline 28 & Boesen et al. [34] & 289 & $64(59-67)$ & $12(8.3-19)$ & $63(46-87)$ \\
\hline 29 & Kasivisvanathan et al.[35] & 500 & 64.4 & $6.62(5.14-9.35)$ & NA \\
\hline 30 & Borkowetz et al. [36] & 214 & $63(40-75)$ & $6.22(1-49)$ & $42(8-270)$ \\
\hline 31 & Hofbauer et al. [37] & 704 & $68(62-73)$ & $7.93(5.97-12.17)$ & $51(40-72)$ \\
\hline \multirow{2}{*}{32} & \multirow{2}{*}{ Costa et al. [38] } & 103 & $64 \pm 7$ & $8 \pm 4.1$ & $55.4 \pm 24.5$ \\
\hline & & 300 & $66 \pm 8$ & $8.6 \pm 11.5$ & $51.6 \pm 23.5$ \\
\hline 33 & Maxeiner et al. [39] & 318 & $68(60-72)$ & $7.14(5.13-10.3)$ & $47(36-65)$ \\
\hline \multirow{3}{*}{34} & \multirow{3}{*}{ Wegelin et al. [40] } & 79 & $64.6 \pm 6.9$ & $11.6 \pm 9.0$ & $45.4 \pm 14.4$ \\
\hline & & 78 & $66.5 \pm 6.3$ & $11.0 \pm 7.1$ & $48.5 \pm 18.1$ \\
\hline & & 77 & $66.0 \pm 5.9$ & $11.0 \pm 9.4$ & $48.3 \pm 20.2$ \\
\hline 35 & van der Leest [41] & 326 & $65(59-68)$ & $6.4(4.6-8.2)$ & $55(41-77)$ \\
\hline \multirow{2}{*}{36} & \multirow{2}{*}{ Elkhoury et al. [42] } & 248 & $65.5 \pm 7.7$ & $6.2 \pm 4.6-8.2$ & NA \\
\hline & & 248 & $65.5 \pm 7.7$ & $6.2 \pm 4.6-8.2$ & NA \\
\hline 37 & Rouviere et al. [43] & 251 & $64(59-68)$ & $6.5(5.6-9.6)$ & $50(38-63)$ \\
\hline
\end{tabular}




\section{MRI-targeted biopsy}

\section{A. Cognitive MRI-targeted biopsy}

The first method of performing targeted MRI guided biopsies to be employed in clinical practice was Cog-TBx. However, due to the increasing availability of MRI-TRUS fusion and in-bore systems, the number of trials that observed the 8 -years inclusion criteria was limited.

Eight studies were selected for our analysis, presenting conflicting results: Wysock, Elkhoury and Castelucci $[7,32,42]$ report superiority of standard systematic biopsy (SBx) compared to Cog-TBx in terms of overall cancer detection rate (CDR) in biopsy naïve patients, while Delongchamps et al. [10] concludes the opposite. Tonttila et al. [8] and Kam et al. [11] presented the non-inferiority of $\operatorname{Cog}-\mathrm{TBx}$ compared to $\mathrm{SBx}$. Also, Cog-TBx presents the lowest performance in $\mathrm{PCa}$ diagnosis when compared to FUS-TBx or IB-TBx, even though it is not statistically significant [40]. Despite these results, Cog-TBx has similar detection rates of csPCa with saturation biopsy, with statistically significant lower number of cores obtained [44]. Association of Cog-TBx with $\mathrm{SBx}$ presents higher accuracy for $\mathrm{PCa}$ and csPCa diagnosis when compared to each technique alone ( $\mathrm{SBx}$ or Cog-TBx) [11,32,42].

All studies reported that targeted biopsy methods were significantly more efficient when compared to SBx regarding cancer core length/core and number of positive cores.

\section{B. MRI/TRUS Fusion targeted biopsy}

Fus-TBx combines the advantages of both CogTBx and IB-TBx, fusing the broad availability of CogTBx with real time visualization of the tumor by magnetic resonance imaging [45].

Twenty-nine of the studies compiled in our analysis compared TBx with the previously listed techniques. In 17 studies, the CDR were similar between the evaluated methods (TBx vs SBx, $\pm 5-10 \%$ ), while 2 studies reported a statistically significant higher detection rate for Fus$\mathrm{TBx}$, with an improvement of at least $10 \%$, compared to SBx $[27,28]$. Only one study favored SBx concerning cancer detection rate [23]. PROFUS Trial reported no statistically significant difference between Cog-TBx and Fus-TBx in terms of CDR, with the mention that FUSTBx provided better histological specimens compared to visual targeting [7]. Several studies demonstrate that FusTBx combined with SBx outperforms both Fus-TBx and SBx alone [34,36,38,39,42,43].

Regarding CDR for csPCa, 20 studies showed higher prevalence of csPCa for the patients biopsied by Fus-TBx, in 8 of them the difference was statistically significant $[18,21,25-28,34,35]$. Even in a multicenter designed trial, Fus-TBx outperformed SBx for the diagnosis of csPCa [35], while the detection rate for is PCa was lower [21,28,31,33-35,39,42,43]. It is necessary to mention that the definition of csPCa varied significantly among the studies. Fus-TBx presents a high performance in reducing statistically significant the diagnosis rate of insignificant PCa compared to SBx $[21,28,31,34,43]$.

Five studies reported upgrading rates when FusTBx was performed compared to SBx, varying between $7.58 \%$ and $35 \%$ (Table III). Siddiqui's trial of 1003 patients, the most exhaustive study published up to date, concluded that Fus-TBx increased the diagnostic rate of high-risk cancers up to $30 \%(\mathrm{p}<0.001)$ and upgrading to intermediate or high-risk groups $(\mathrm{p}<0.001)$, compared to SBx [21].

In terms of diagnostic efficiency, Radtke et al. showed that even if saturation biopsy outruled Fus-TBx from the perspective of CDR, it still needs twice more cores to detect $1 \mathrm{GS} \geq 7$ ( 7.4 cores vs 3.4 cores) [23]. On the other hand, PRECISION multicenter trial reported a statistical significant better performance of Fus-TBx for CDR and diagnostic efficiency, while similar efficiency was observed in several other trials $[12,16,26]$.

\section{In-Bore MRI targeted biopsy (IB-TBx)}

In-Bore MRI targeted biopsy is considered to be the most precise method. In the present paper, we included 7 studies: 4 comparing IB-TBx to SBx while 3 IB-TBx to Fus-TBx. Transrectal approach was used in all evaluated studies.

The over-all CDR for $\mathrm{PCa}$ varied significantly from $37 \%$ to $69.9 \%$, with some authors reporting better diagnosis rates for IB-TBx compared to SBx $[12,14]$. IBTBx presented higher CDR compared to Fus-TBx or CogTBx, although not statistically significant $[13,21,34]$.

Reviewing $\mathrm{CDR}$ for csPCa, all authors showed that IB-TBx has a higher efficiency, compared to standard randomized biopsy $[12-15,38,41]$, but similar to the FusTBx [15]. Also, IB-TBx identifies a statistically significant lower percentage of insignificant $\mathrm{PCa}$ when compared to SBx $[14,41]$, upgrading $7 \%$ to $32 \%$ of cases diagnosed as no cancer or low risk PCa by SBx $[13,14,41]$. It presents a $9-10 \%$ risk of missing csPCa $[13,41]$, depending upon the lesion dimension and location [46].

Pokorny et al. were the only ones to evaluate the two modalities from the perspective of sensitivity, specificity, positive negative predictive value, for each category IB-TBx outperforming SBx [14].

Regarding tumor features, IB-TBx presented higher efficacy in the number of biopsy cores needed to detect csPCa $[14,15,41]$. Average percentage of cancer core length was statistically higher for IB-TBx $(p<0,001)$ [14], but the differences fade when Fus-TBx is associated with SBx [15]. 


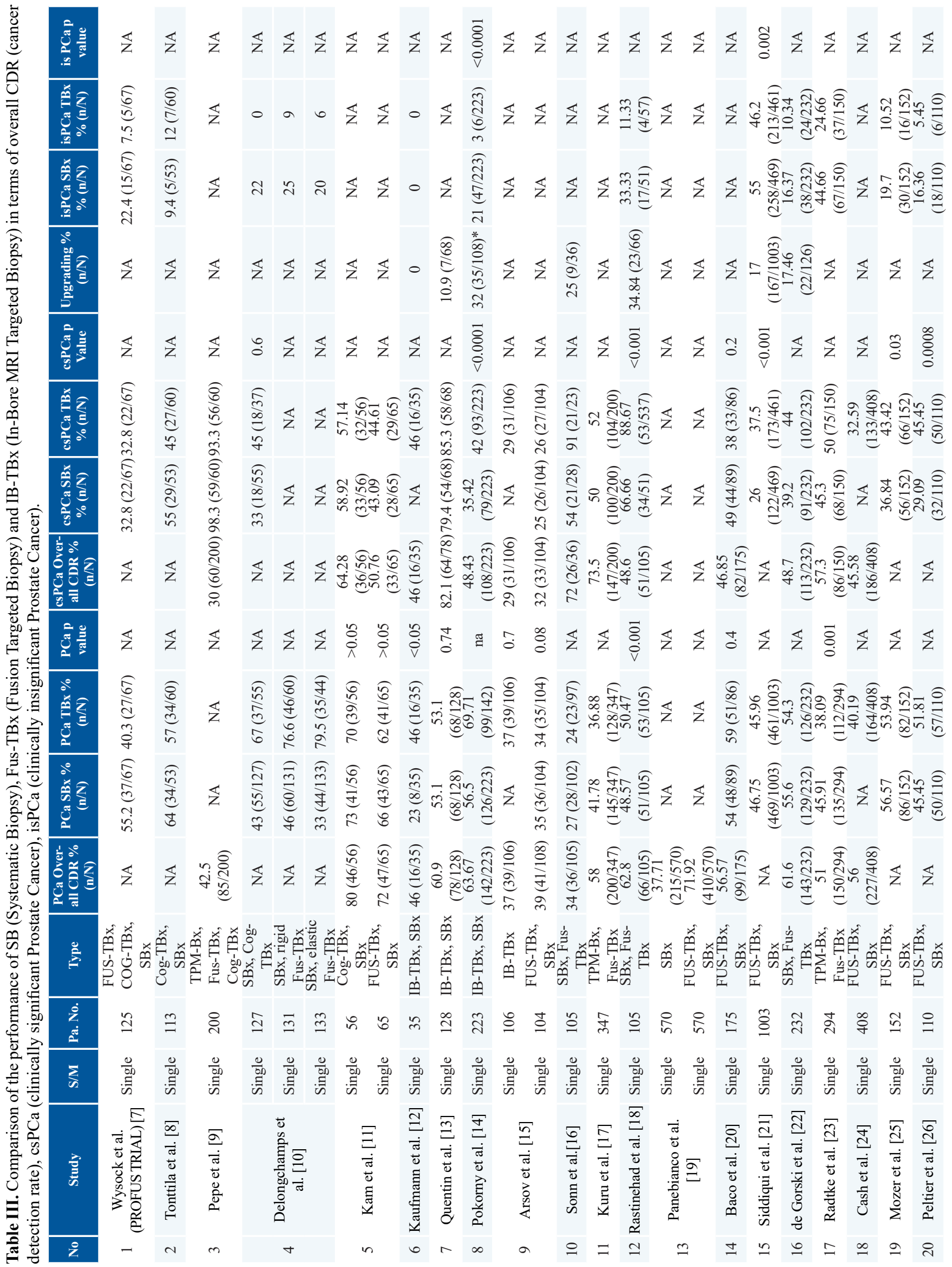




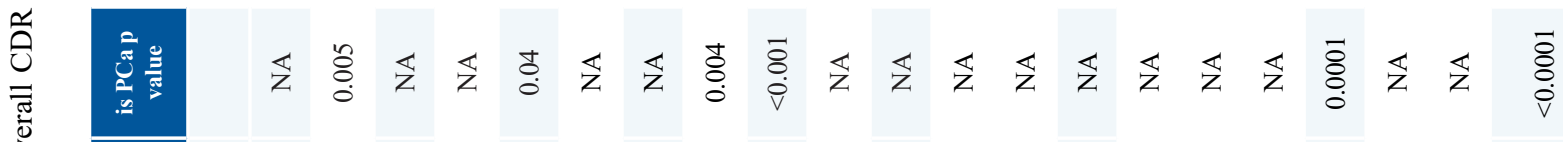

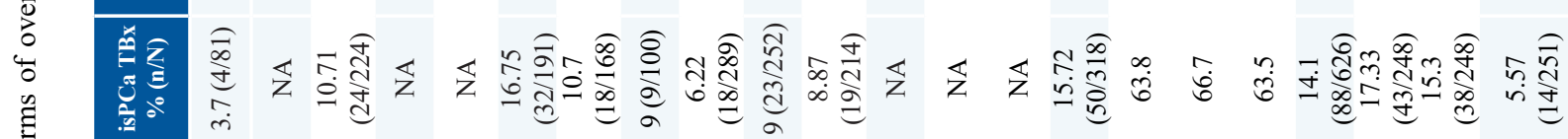

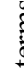

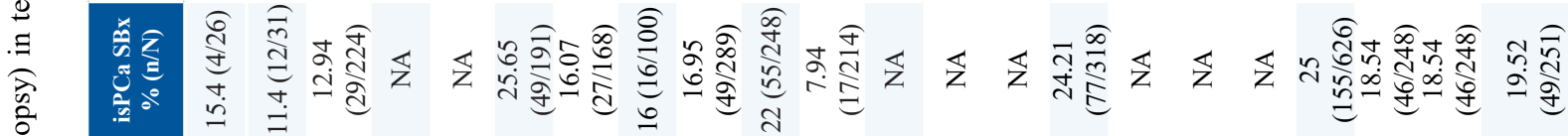

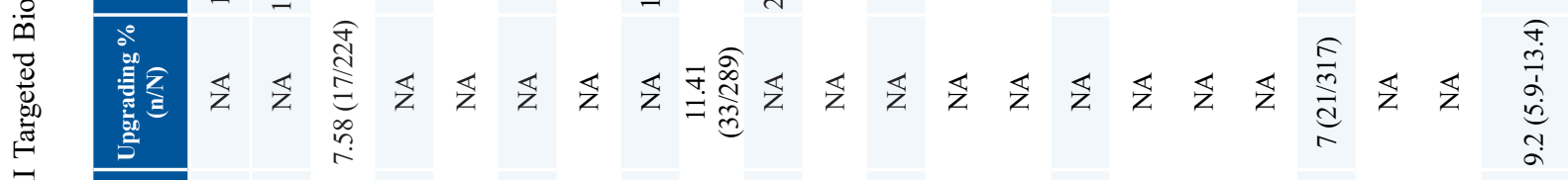

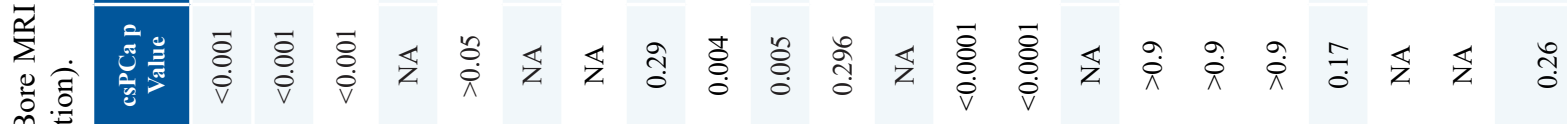

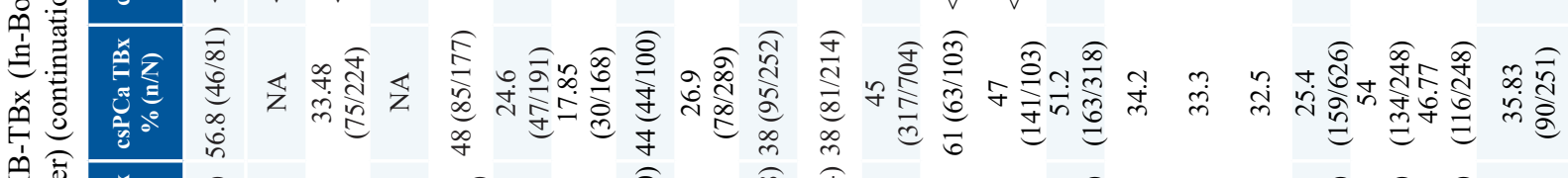

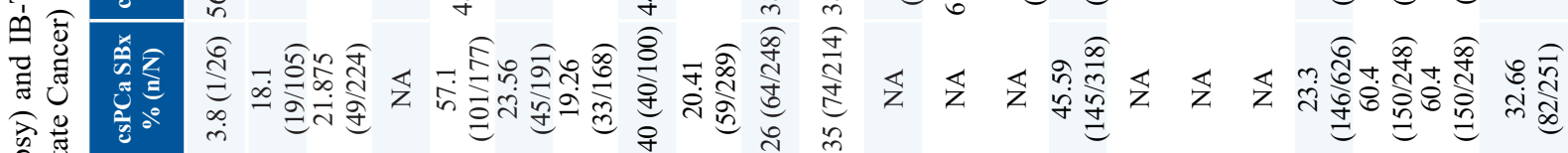

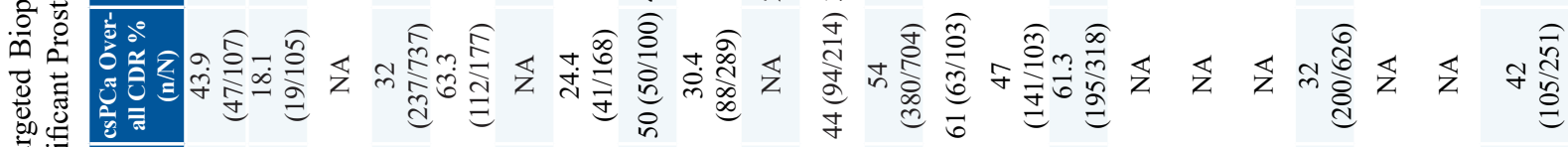

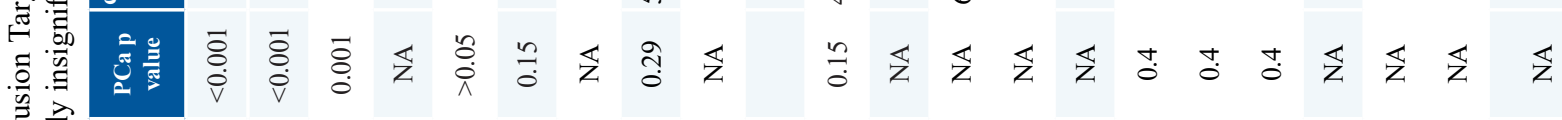

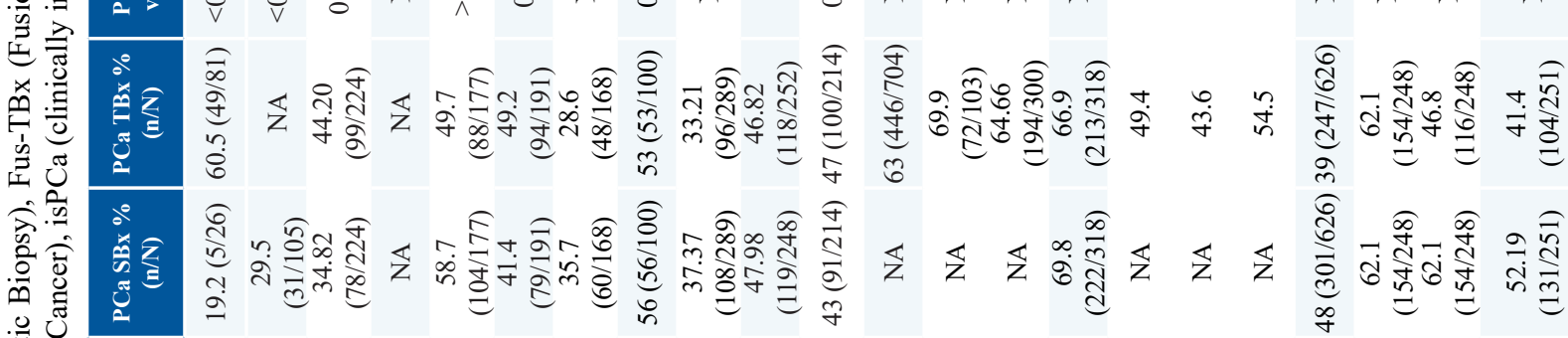

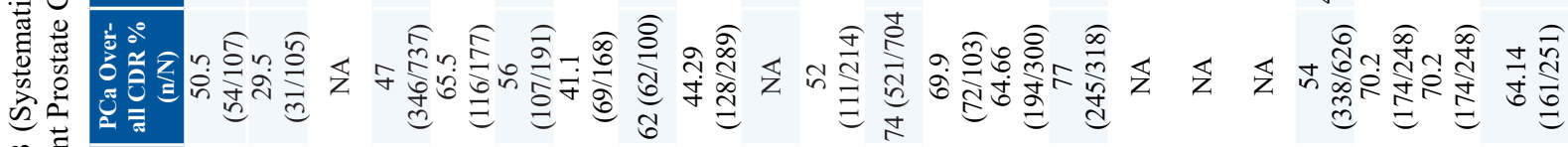

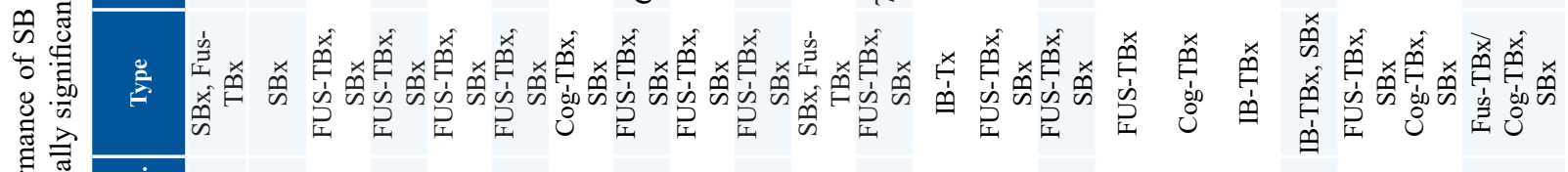

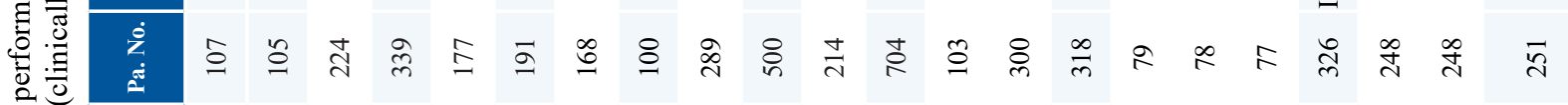

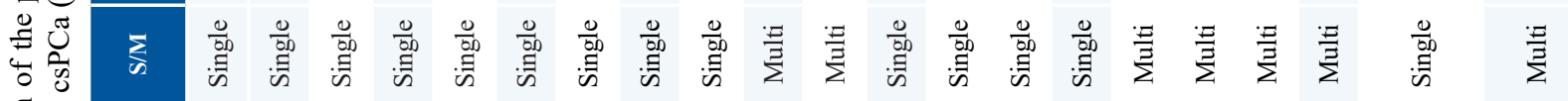

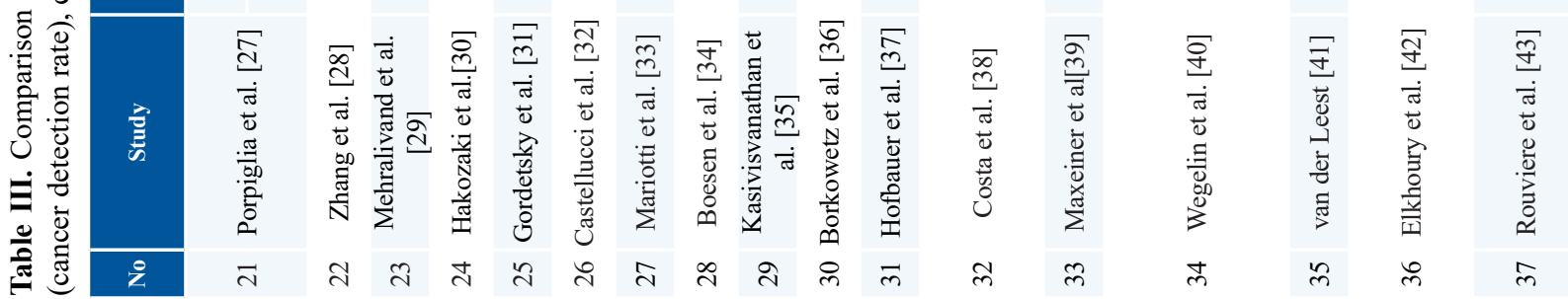




\section{Discussion}

1. TBx impact on diagnosis and treatment management

Treatment options for non-metastatic PCa include active surveillance, focal therapy, radiation therapy and radical prostatectomy. The therapeutic decision is mainly based on the histopathological examination, provided by the prostate biopsy, randomized or targeted. Multiparametric MRI provides improved diagnosis rate for csPC and staging information, which are the basis for an informed treatment choice and future planning in case of surgical treatment or focal therapies [19].

\section{A. TBx and prostate cancer diagnosis}

- Malignant vs. benign

The cumulative risk of prostate cancer diagnosis increases with each repeated biopsy $(68 \%$ after four biopsies), with $38 \%$ of patients requiring a second biopsy after 5 years from the first systematic biopsy [47]. This risk may be lowered by mpMRI and targeted biopsy, taking into consideration that in our analysis TBx outperformed SBx in the majority of studies. Moreover, there is no study to present a statistically significant higher CDR for the SBx, while it was observed that both in high level single institutions and multicenter (PRECISION, FUTURE) trials it is possible to achieve a higher cancer detection rate by TBx. Association of the two biopsy techniques may overcome the current limitations of targeted biopsy by improving both overall $\mathrm{PCa}$ and csPCa cancer detection rates. Dell'Oglio et al. emphasized the importance of systematic biopsy to limit the missed csPCa cases by targeted biopsy [48].

Multiparametric MRI (mpMRI) and the subsequent TBx need further improvement, due to a small percentage of prostate cancers being missed with these methods, while properly detected in SBx cases $[14,35]$.

To conclude, even if Cog-TBx is the only available option, mpMRI should be performed prior to the biopsy, to increase the accuracy of diagnosis.

- CsPCa vs isPCa

Assessing the patient in the correct risk category is mandatory in order to decide further treatment. The most important limitation encountered in daily practice is the high inter-studies variability regarding the definition of csPCa (Table I). However, TBx offers more accurate diagnosis of csPCa, especially when Fus-TBx or IB-TBx are performed (Table III) [14,27,28]. Moreover, TBx presents lower detection rate of low risk PCa compared to SBx (Table III).

Cribriform and intraductal prostate carcinoma are included in the clinically significant prostate cancers group, having an aggressive evolution. There are conflicting results in terms of mpMRI performance of identifying these lesions and which biopsy type is more adequate $[41,42]$. The latest trial to address the issue demonstrated that FusTBx significantly outperformed SBx [50].

\section{- Location of the lesion}

Lesion localization is an important factor to be taken into consideration, being proved that in case of previous negative biopsy there is a higher probability that the lesion is located in the anterior region of the prostate, which also harbors more frequently csPCa [51]. In a retrospective analysis of 499 patients, half of the 241 anterior lesions identified on mpMRI were positive for PCa, Fus-TBx outperforming SBx in diagnosing PCa located anteriorly $(\mathrm{p}=0.001)[52]$.

On the other hand, there are differences between the targeted and systematic biopsies in reaching different prostate zones. Both TBx and SBx may miss apical lesions [46]. IB-TBx potentially misses more csPCa located in dorsolateral and apical segments of the prostate, while SBx tends to miss csPCa in the anterior, anterior midprostate and anterior apex [46].

- Limitations

Despite the fact that the initial mpMRI does not identify any lesion, in up to $26 \%$ of patients csPCa may still be present [53]. Standardization and improvement of mpMRI interpretation (PI-RADS v2, v2.1) increased the negative predictive value of mpMRI, up to $82.4 \%$ for overall cancer and $88.1 \%$ for csPCa [54]. A negative TBx cannot exclude the presence of csPCa, as $46 \%$ of the highly suspicious lesions described on MRI can harbor csPCa [55], while repeated Fus-TBx reveals clinically significant discordance with the initial TBx in more than half of the cases [56].

Two possible reasons may explain the percentage of misdiagnosis for targeted biopsy, as reported by Cash et al: firstly, the inaccurate PIRADS Score classification, inexperienced readers having a tendency of reporting higher initial PIRADS score, in up to $39 \%$ of lesions [24]. Secondly, TBx failure may be due to prostate movement/ deformation by the transrectal ultrasound probe, patient movement, incorrect image registration or mismatch of image planes, but no significant differences were shown by elastic registration or real-time sensor based image registration platforms [57].

The accuracy of three-dimensional MRI/US registration associates a mean target error of 2.4-2.5 mm [58]. An in-vivo experiment showed that in daily practice, co-registration error may be up to $5.6 \mathrm{~mm}$, with the highest errors encountered at the apex of the gland [59]. Despite higher csPCa detection rate when elastic registration was performed, it was not statistically significant different to the rigid one [60]. The skills required to perform TBx improve in time as revealed in a cohort of 1330 patients that underwent Fus-TBx when csPCa detection rate raised by $26 \%$, between the initial and last 190 cases [56].

Moreover, Halstuch et al. showed that larger prostates and right sided biopsy may significantly induce a needle tip deflection, which may account for targeted biopsy error [61]. Despite that prostate volume alters 
the accuracy of the biopsy, it was observed that FusTBx performs better than SBx in larger prostates [22]. In the multivariate analysis, Mozer et al. reported that the probability for detecting csPCa increased in cases of lower volume prostates and larger MRI lesions [25].

\section{B. TBx and prostate cancer treatment}

- PCa grading

Prostate cancer staging and grading are essential for the choice between active surveillance, focal therapy or radical therapy. Despite the PCa multifocality, in up $97.5 \%$ of cases the index tumor is the one with the largest volume [53], and several studies reported MRI detection rate around $92 \%[57,58]$. MRI performance in identifying index tumors $>0.5 \mathrm{~mL}$ varied among $86 \%-92 \%[53,63]$.

SBx is responsible for up to $30 \%$ of Gleason Score (GS) incorrect classification [64]. Performing saturation biopsy does not significantly improve rate of correct GS [65], while the associated risks of the procedure are wellknown. There is better correlation between the GS of biopsy and prostatectomy specimen when Fus-TBx is performed compared to SBx, both in terms of primary and secondary Gleason grade pattern [66].

From the perspective of GS down-grading, Porpiglia et al. reported in a retrospective analysis of 683 cases, that up to one-third of the patients diagnosed by SBx with Gleason 8 were down-graded. Down-grading has a strong impact on the chosen therapy, a tendency to recommend radiation therapy with long-term androgen deprivation for patients with GS 8 or higher has been observed [66].

- Active surveillance (AS) selection and reevaluation

MpMRI is a useful tool in selecting patients for AS due to the high negative predictive value for csPCa [67]. mpMRI provides additional information compared to other imagistic examination. Firstly, it evaluates the extraprostatic extension (EPE), being observed that in those with extraprostatic extension the risk of $\mathrm{csPCa}$ is 12 times higher, therefore a more aggressive option is chosen [68]. Secondly, targeted biopsy based on mpMRI reclassified up $10 \%$ of patients considered eligible for AS based on SBx [69], with Fus-TBx upgrading up to $24 \%$ of SBx results [70].

Multiparametric MRI can play a role in the reevaluation of patients under AS, with the possibility to avoid re-biopsy in patients with PIRADS $\leq 3$ lesions and no sign of extra-prostatic extension [68]. PIRADS score is an independent predictor of csPCa in patients benefiting from AS [71]. However, in case of repeated negative mpMRI, biopsy cannot be ruled out because a low risk of csPCa still persists [68]. TBx performs better in the followup evaluation of patients under AS compared with SBx $[65,68]$.

- MRI/TRUS Fusion impact on focal therapy

MRI/TRUS Fusion technology not only guides the biopsy, but also focal therapies. MRI/TRUS Fusion technology meets the precision requirement, providing more accessible environment to perform the focal therapy in comparison with the MRI gantry.

Despite an initial pilot study with a $100 \%$ recurrence free rate at four weeks after the MRI/TRUS Fusion HIFU [73], another prospective trial with double the number of patients and 12 month reevaluation landmark revealed a recurrence rate of $40 \%$ and established that the technique needs further evaluation and improvement [74].

Other focal therapies used were MRI/TRUS Fusion focal cryotherapy, which showed no evident imagistic recurrence, not leaving the possibility of bioptic reevaluation [75], and MRI/TRUS Fusion focal laser ablation which in the pilot study presented no recurrence at the six month rebiopsy [76].

\section{TBx cost-effectiveness}

The associated higher costs for targeted biopsy (mpMRI, biopsy platform, materials) represent the main argument against its wide employment [77]. PROMIS Trial suggested that mpMRI prior to biopsy has several advantages with possible impact into the healthcare costs, such as avoiding prostate biopsy in up to a quarter of patients, for those with no MRI or low score PIRADS lesion [78]. As shown by our analysis, TBx lowers clinically insignificant cancer diagnosis rate, which will decrease the overtreatment observed and its associated side-effects. A higher detection of clinically significant $\mathrm{PCa}$, from the initial biopsy lowers the number of further necessary investigation with patients benefitting from the correct treatment from the beginning [78]. PRECISION Trial showed that the number of patients with persistent $\mathrm{PCa}$ suspicion who needed further investigation was 5 times higher for those who were evaluated by SBx [35].

Venderick et al. performed a computer simulation for biopsy naïve population, which showed that fusion biopsy is cost-effective in the context of two different healthcare systems [79]. Another similar study based on the PROMIS Trial data, showed the same cost-effectiveness of using mpMRI and TBx [80].

\section{Future improvement directions}

mpMRI interpretation training and reproducibility is crucial in establishing TBx as standard of care. PROMIS and PRECISION Trials supported the collaboration with experienced radiologists, who also benefitted from training in the pilot phase of the study $[35,78]$. The key to achieve higher sensibility and specificity regarding mpMRI diagnosis of csPCa lies within the improvement brought by PIRADS v2 and v2.1, associated with efficient training for the radiologist. Also, the performance in terms of CDR is correlated with the learning curve [37].

Collaboration between radiologists and urologists is essential. In our analysis in 21 out of the 37 studies, TBx was performed solely by the urologist. It is observed that the definition of TBx between the two specialists may differ 
in up to $23 \%$ cases, with urologists identifying more lesions and with larger volumes in disagreement with PIRADS criteria [81]. A pilot study using a mpMRI case-based ibook for urologists demonstrated a statistically significant improvement in mean score test after book review $(37 \%$ to $57 \%, \mathrm{p}=0.0039$ ) [82]. Therefore, urologist training in mpMRI reading is essential to overcome errors such as lesion choice, target lesion dimensions [81].

Having in mind the persistent low number of csPCa missed by mpMRI and TBx, it is necessary to improve the existent technology by better identification and visualization of suspect lesions using MRI spectroscopy and in vivo metabolic changes observed in prostate cancer [83]. Better performance is needed in terms of higher accuracy when performing registration of the ultrasonography and MRI, which may be acquired using co-registrations markers such as fiducial ones, visible on MRI and US, guiding both biopsy and focal therapy [59].

There are further studies needed in order to evaluate whether factors such as PIRADS score, prostate dimension, number of lesions, location and dimension, anatomic particularities and technique biases can influence the urologist into choosing the right targeted biopsy technique for the patient.

\section{Conclusion}

Targeted biopsy is an emerging and developing technique which offers the needed improvements in diagnosing clinically significant prostate cancer and lowers the incidence of insignificant one, providing more accurate selection of the patients for active surveillance and focal therapies. No significant differences between the MRI targeting techniques have been observed, the key for an accurate diagnosis being the individualization of the biopsy technique according to the particularities of each patient.

\section{Acknowledgement}

This work was supported by the Iuliu Hatieganu University of Medicine and Pharmacy, Cluj-Napoca, Romania through the Doctoral Research Program grant 2461/9/17.01.2020.

\section{References}

1. Siegel RL, Miller KD, Jemal A. Cancer statistics, 2018. CA Cancer J Clin. 2018;68:7-30.

2. Malvezzi M, Carioli G, Bertuccio P, Boffetta P, Levi F, Vecchia $\mathrm{C} \mathrm{La}$, et al. European cancer mortality predictions for the year 2018 with focus on colorectal cancer. Ann Oncol. 2018;29:1016-1022.

3. Mottet N, van den Bergh RCN, Briers E, Bourke L, Cornford P, De Santis M et al. EAU - ESTRO - ESUR - SIOG Guidelines on Prostate Cancer.Eur Assoc Urol Guide 2018. Available from: https://uroweb.org/wp-content/uploads/ EAU-ESUR-ESTRO-SIOG-Guidelines-on-Prostate-Cancer-
large-text-V2.pdf

4. Hodge KK, McNeal JE, Stamey TA. Ultrasound guided transrectal core biopsies of the palpably abnormal prostate. $\mathrm{J}$ Urol. 1989;142:66-70.

5. Lee DJ, Recabal P, Sjoberg DD, Thong A, Lee JK, Eastham JA, et al. Comparative Effectiveness of Targeted Prostate Biopsy Using Magnetic Resonance Imaging Ultrasound Fusion Software and Visual Targeting: a Prospective Study. J Urol. 2016;196:697-702.

6. Mottet N, van den Bergh RCN, Briers E, Cornford P, De Santis M, Fanti S, et al. EAU-EANM-ESTRO-ESURSIOG Guidelines on Prostate Cancer. European Association of Urology; 2019. Available from: https://uroweb.org/ wp-content/uploads/EAU-EANM-ESUR-ESTRO-SIOGGuidelines-on-Prostate-Cancer-2019.pdf

7. Wysock JS, Rosenkrantz AB, Huang WC, Stifelman MD, Lepor H, Deng FM, et al. A prospective, blinded comparison of magnetic resonance (MR) imaging-ultrasound fusion and visual estimation in the performance of MR-targeted prostate biopsy: The PROFUS trial. Eur Urol. 2014;66:343-351.

8. Tonttila PP, Lantto J, Pääkkö E, Piippo U, Kauppila S, Lammentausta E, et al. Prebiopsy Multiparametric Magnetic Resonance Imaging for Prostate Cancer Diagnosis in Biopsy-naive Men with Suspected Prostate Cancer Based on Elevated Prostate-specific Antigen Values: Results from a Randomized Prospective Blinded Controlled Trial. Eur Urol. 2016;69:419-425.

9. Pepe P, Garufi A, Priolo G, Pennisi M. Can MRI/TRUS fusion targeted biopsy replace saturation prostate biopsy in the re-evaluation of men in active surveillance? World $\mathbf{J}$ Urol. 2016;34:1249-1253.

10. Delongchamps NB, Peyromaure M, Schull A, Beuvon F, Bouazza N, Flam T, et al. Prebiopsy magnetic resonance imaging and prostate cancer detection: Comparison of random and targeted biopsies. J Urol. 2013;189:493-499.

11. Kam J, Yuminaga Y, Kim R, Aluwihare K, Macneil F, Ouyang R, et al. Does magnetic resonance imaging-guided biopsy improve prostate cancer detection? A comparison of systematic, cognitive fusion and ultrasound fusion prostate biopsy. Prostate Int. 2018;6:88-93.

12. Kaufmann S, Kruck S, Kramer U, Gatidis S, Stenzl A, Roethke M, et al. Direct comparison of targeted MRI-guided biopsy with systematic transrectal ultrasound-guided biopsy in patients with previous negative prostate biopsies. Urol Int. 2015;94:319-325.

13. Quentin M, Blondin D, Arsov C, Schimmöller L, Hiester A, Godehardt E, et al. Prospective evaluation of magnetic resonance imaging guided in-bore prostate biopsy versus systematic transrectal ultrasound guided prostate biopsy in biopsy naïve men with elevated prostate specific antigen. J Urol. 2014;192:1374-1379.

14. Pokorny MR, De Rooij M, Duncan E, Schröder FH, Parkinson $\mathrm{R}$, Barentsz JO, et al. Prospective study of diagnostic accuracy comparing prostate cancer detection by transrectal ultrasound-guided biopsy versus magnetic resonance (MR) imaging with subsequent mr-guided biopsy in men without previous prostate biopsies. Eur Urol. 2014;66:22-29. 
15. Arsov C, Rabenalt R, Blondin D, Quentin M, Hiester A, Godehardt E, et al. Prospective randomized trial comparing magnetic resonance imaging (MRI)-guided in-bore biopsy to MRI-ultrasound fusion and transrectal ultrasound-guided prostate biopsy in patients with prior negative biopsies. Eur Urol. 2015;68:713-720.

16. Sonn GA, Chang E, Natarajan S, Margolis DJ, MacAiran $\mathrm{M}$, Lieu $\mathrm{P}$, et al. Value of targeted prostate biopsy using magnetic resonance-ultrasound fusion in men with prior negative biopsy and elevated prostate-specific antigen. Eur Urol. 2014;65:809-815.

17. Kuru TH, Roethke MC, Seidenader J, Simpfendörfer T, Boxler S, Alammar K, et al. Critical evaluation of magnetic resonance imaging targeted, transrectal ultrasound guided transperineal fusion biopsy for detection of prostate cancer. $\mathrm{J}$ Urol. 2013;190:1380-1386.

18. Rastinehad AR, Turkbey B, Salami SS, Yaskiv O, George AK, Fakhoury M, et al. Improving detection of clinically significant prostate cancer: Magnetic resonance imaging/ transrectal ultrasound fusion guided prostate biopsy. J Urol. 2014;191:1749-1754.

19. Panebianco V, Barchetti F, Sciarra A, Ciardi A, Indino EL, Papalia R, et al. Multiparametric magnetic resonance imaging vs. standard care in men being evaluated for prostate cancer: A randomized study. Urol Oncol. 2015;33:17.e1-17.e7.

20. Baco E, Rud E, Eri LM, Moen G, Vlatkovic L, Svindland A, et al. A Randomized Controlled Trial to Assess and Compare the Outcomes of Two-core Prostate Biopsy Guided by Fused Magnetic Resonance and Transrectal Ultrasound Images and Traditional 12-core Systematic Biopsy. Eur Urol. 2016;69:149-156.

21. Siddiqui MM, Rais-Bahrami S, Turkbey B, George AK, Rothwax J, Shakir N, et al. Comparison of MR/ultrasound fusion-guided biopsy with ultrasound-guided biopsy for the diagnosis of prostate cancer. JAMA. 2015;313:390-397.

22. de Gorski A, Rouprêt M, Peyronnet B, Le Cossec C, Granger B, Comperat E, et al. Accuracy of magnetic resonance imaging/ultrasound fusion targeted biopsies to diagnose clinically significant prostate cancer in enlarged compared to smaller prostates. J Urol. 2015;194:669-673.

23. Radtke JP, Kuru TH, Boxler S, Alt CD, Popeneciu IV, Huettenbrink C, et al. Comparative Analysis of Transperineal Template Saturation Prostate Biopsy Versus Magnetic Resonance Imaging Targeted Biopsy with Magnetic Resonance Imaging-Ultrasound Fusion Guidance. J Urol. 2015;193:87-94.

24. Cash H, Maxeiner A, Stephan C, Fischer T, Durmus T, Holzmann $\mathrm{J}$, et al. The detection of significant prostate cancer is correlated with the Prostate Imaging Reporting and Data System (PI-RADS) in MRI/transrectal ultrasound fusion biopsy. World J Urol. 2016;34:525-532.

25. Mozer P, Rouprêt M, Le Cossec C, Granger B, Comperat E, de Gorski A, et al. First round of targeted biopsies using magnetic resonance imaging/ultrasonography fusion compared with conventional transrectal ultrasonographyguided biopsies for the diagnosis of localised prostate cancer. BJU Int. 2015;115:50-57.

26. Peltier A, Aoun F, Lemort M, Kwizera F, Paesmans M,
Van Velthoven R. MRI-targeted biopsies versus systematic transrectal ultrasound guided biopsies for the diagnosis of localized prostate cancer in biopsy naïve men. Biomed Res Int. 2015;2015:571708.

27. Porpiglia F, Manfredi M, Mele F, Cossu M, Bollito E, Veltri A, et al. Diagnostic Pathway with Multiparametric Magnetic Resonance Imaging Versus Standard Pathway: Results from a Randomized Prospective Study in Biopsy-naïve Patients with Suspected Prostate Cancer. Eur Urol. 2017;72:282-288.

28. Zhang Q, Wang W, Zhang B, Shi J, Fu Y, Li D, et al. Comparison of free-hand transperineal mpMRI/TRUS fusion-guided biopsy with transperineal 12-core systematic biopsy for the diagnosis of prostate cancer: a single-center prospective study in China. Int Urol Nephrol. 2017;49:439448.

29. Mehralivand S, Bednarova S, Shih JH, Mertan FV, Gaur $\mathrm{S}$, Merino MJ, et al. Prospective Evaluation of PI-RADSTM Version 2 Using the International Society of Urological Pathology Prostate Cancer Grade Group System. J Urol. 2017;198:583-590.

30. Hakozaki Y, Matsushima H, Kumagai J, Murata T, Masuda $\mathrm{T}$, Hirai $\mathrm{Y}$, et al. A prospective study of magnetic resonance imaging and ultrasonography (MRI/US)-fusion targeted biopsy and concurrent systematic transperineal biopsy with the average of 18 -cores to detect clinically significant prostate cancer. BMC Urol. 2017;17:117.

31. Gordetsky JB, Thomas JV, Nix JW, Rais-Bahrami S. Higher prostate cancer grade groups are detected in patients undergoing multiparametric MRI-targeted biopsy compared with standard biopsy. Am J Surg Pathol. 2017;41:101-105.

32. Castellucci R, Linares Quevedo AI, Sánchez Gómez FJ, Díez Rodríguez J, Cogorno L, Cogollos Acuña I, et al. Prospective nonrandomized study of diagnostic accuracy comparing prostate cancer detection by transrectal ultrasound-guided biopsy to magnetic resonance imaging with subsequent MRI-guided biopsy in biopsy-naïve patients. Minerva Urol Nefrol. 2017;69:589-595.

33. Mariotti GC, Falsarella PM, Garcia RG, Queiroz MRG, Lemos GC, Baroni RH. Incremental diagnostic value of targeted biopsy using mpMRI-TRUS fusion versus 14-fragments prostatic biopsy: a prospective controlled study. Eur Radiol. 2018;28:11-16.

34. Boesen L, Nørgaard N, Løgager V, Balslev I, Thomsen HS. Multiparametric MRI in men with clinical suspicion of prostate cancer undergoing repeat biopsy: a prospective comparison with clinical findings and histopathology. Acta Radiol. 2018;59:371-380.

35. Kasivisvanathan V, Rannikko AS, Borghi M, Panebianco V, Mynderse LA, Vaarala MH, et al. MRI-targeted or standard biopsy for prostate-cancer diagnosis (Precision Study). N Engl J Med. 2018;378:1767-1677.

36. Borkowetz A, Renner T, Platzek I, Toma M, Herout R, Baunacke $\mathrm{M}$, et al. Evaluation of Magnetic Resonance Imaging/Ultrasound-Fusion Biopsy in Patients with LowRisk Prostate Cancer under Active Surveillance Undergoing Surveillance Biopsy. Urol Int. 2018;100:155-163.

37. Hofbauer SL, Maxeiner A, Kittner B, Heckmann R, Reimann $\mathrm{M}$, Wiemer L, et al. Validation of Prostate Imaging Reporting 
and Data System Version 2 for the Detection of Prostate Cancer. J Urol. 2018; 200:767-773.

38. Costa DN, Goldberg K, Leon AD, Lotan Y, Xi Y, Aziz M, et al. Magnetic Resonance Imaging-guided In-bore and Magnetic Resonance Imaging-transrectal Ultrasound Fusion Targeted Prostate Biopsies: An Adjusted Comparison of Clinically Significant Prostate Cancer Detection Rate. Eur Urol Oncol. 2019;2:397-404.

39. Maxeiner A, Kittner B, Blobel C, Wiemer L, Hofbauer SL, Fischer T, et al. Primary magnetic resonance imaging/ ultrasonography fusion-guided biopsy of the prostate. BJU Int. 2018;122:211-218.

40. Wegelin O, Exterkate L, van der Leest M, Kummer JA, Vreuls W, de Bruin PC, et al. The FUTURE Trial: A Multicenter Randomised Controlled Trial on Target Biopsy Techniques Based on Magnetic Resonance Imaging in the Diagnosis of Prostate Cancer in Patients with Prior Negative Biopsies. Eur Urol. 2019;75:582-590.

41. van der Leest M, Cornel E, Israël B, Hendriks R, Padhani AR, Hoogenboom M, et al. Head-to-head Comparison of Transrectal Ultrasound-guided Prostate Biopsy Versus Multiparametric Prostate Resonance Imaging with Subsequent Magnetic Resonance-guided Biopsy in Biopsynaïve Men with Elevated Prostate-specific Antigen: A Large Prospective Multicenter Clinical Study. Eur Urol. 2019;75:570-578.

42. Elkhoury FF, Felker ER, Kwan L, Sisk AE, Delfin M, Natarajan S, et al. Comparison of Targeted vs Systematic Prostate Biopsy in Men Who Are Biopsy Naive: The Prospective Assessment of Image Registration in the Diagnosis of Prostate Cancer (PAIREDCAP) Study. JAMA Surg. 2019;154:811-818.

43. Rouvière $\mathrm{O}$, Puech $\mathrm{P}$, Renard-Penna R, Claudon M, Roy $\mathrm{C}$, Mège-Lechevallier $\mathrm{F}$, et al. Use of prostate systematic and targeted biopsy on the basis of multiparametric MRI in biopsy-naive patients (MRI-FIRST): a prospective, multicentre, paired diagnostic study. Lancet Oncol. 2019;20:100-109.

44. Pepe P, Garufi A, Priolo G, Pennisi M. Transperineal Versus Transrectal MRI/TRUS Fusion Targeted Biopsy: Detection Rate of Clinically Significant Prostate Cancer. Clin Genitourin Cancer. 2017;15:e33-e36.

45. Schoots IG, Roobol MJ, Nieboer D, Bangma CH, Steyerberg EW, Hunink MG. Magnetic Resonance Imaging-targeted Biopsy May Enhance the Diagnostic Accuracy of Significant Prostate Cancer Detection Compared to Standard Transrectal Ultrasound-guided Biopsy: A Systematic Review and Metaanalysis. Eur Urol. 2015;68:438-450.

46. Schouten MG, van der Leest M, Pokorny M, Hoogenboom M, Barentsz JO, Thompson LC, et al. Why and Where do We Miss Significant Prostate Cancer with Multi-parametric Magnetic Resonance Imaging followed by Magnetic Resonance-guided and Transrectal Ultrasound-guided Biopsy in Biopsy-naïve Men? Eur Urol. 2017;71:896-903.

47. Welch HG, Fisher ES, Gottlieb DJ, Barry MJ. Detection of prostate cancer via biopsy in the medicare-SEER population during the PSA era. J Natl Cancer Inst. 2007;99:1395-1400.

48. Dell'Oglio P, Stabile A, Soligo M, Brembilla G, Esposito
A, Gandaglia G, et al. There is no way to avoid systematic prostate biopsies in addition to multiparametric magnetic resonance imaging targeted biopsies. Eur Urol Oncol. 2020;3:112-118

49. Downes MR, Gibson E, Sykes J, Haider M, van der Kwast TH, Ward A. Determination of the Association Between T2weighted MRI and Gleason Sub-pattern: A Proof of Principle Study. Acad Radiol. 2016;23:1412-1421.

50. Prendeville S, Gertner M, Maganti M, Pintilie M, Perlis N, Toi A, et al. Role of Magnetic Resonance Imaging Targeted Biopsy in Detection of Prostate Cancer Harboring Adverse Pathological Features of Intraductal Carcinoma and Invasive Cribriform Carcinoma. J Urol. 2018;200:104-113.

51. Leyh-Bannurah SR, Kachanov M, Beyersdorff D, Preisser F, Tilki D, Fisch M, et al. Anterior Localization of Prostate Cancer Suspicious Lesions in 1,161 Patients Undergoing Magnetic Resonance Imaging/Ultrasound Fusion Guided Targeted Biopsies. J Urol. 2018;200:1035-1040.

52. Volkin D, Turkbey B, Hoang AN, Rais-Bahrami S, Yerram $\mathrm{N}$, Walton-Diaz A, et al. Multiparametric magnetic resonance imaging (MRI) and subsequent MRI/ultrasonography fusionguided biopsy increase the detection of anteriorly located prostate cancers. BJU Int. 2014;114:E43-E49.

53. Rud E, Klotz D, Rennesund K, Baco E, Berge V, Lien D, et al. Detection of the index tumour and tumour volume in prostate cancer using T2-weighted and diffusion-weighted magnetic resonance imaging (MRI) alone. BJU Int. 2014;114:E32E42.

54. Moldovan PC, Van den Broeck T, Sylvester R, Marconi $\mathrm{L}$, Bellmunt J, van den Bergh RCN, et al. What Is the Negative Predictive Value of Multiparametric Magnetic Resonance Imaging in Excluding Prostate Cancer at Biopsy? A Systematic Review and Meta-analysis from the European Association of Urology Prostate Cancer Guidelines Panel. Eur Urol. 2017;72:250-266.

55. Costa DN, Kay FU, Pedrosa I, Kolski L, Lotan Y, Roehrborn $\mathrm{CG}$, et al. An initial negative round of targeted biopsies in men with highly suspicious multiparametric magnetic resonance findings does not exclude clinically significant prostate cancer-Preliminary experience. Urol Oncol Semin Orig Investig. 2017;35:149.e15-149.e21.

56. Meng X, Rosenkrantz AB, Huang R, Deng F, Wysock JS, Bjurlin $\mathrm{M}$, et al. The institutional learning curve of Magnetic Resonance Imaging-Ultrasound Fusion-Targeted Prostate Biopsy: Temporal improvements in cancer detection over 4 years. J Urol. 2018; 200:1022-1029.

57. Giganti F, Moore CM. A critical comparison of techniques for MRI-targeted biopsy of the prostate. Transl Androl Urol. 2017;6:432-443.

58. De Silva T, Fenster A, Cool DW, Gardi L, Romagnoli C, Samarabandu J, et al. 2D-3D rigid registration to compensate for prostate motion during 3D TRUS-guided biopsy. Med Phys. 2013;40:022904.

59. Moldovan P, Udrescu C, Ravier E, Souchon R, Rabilloud $\mathrm{M}$, Bratan F, et al. Accuracy of elastic fusion of prostate magnetic resonance and transrectal ultrasound images under routine conditions: A prospective multi-operator study. PLoS One. 2016;11:e0169120.

60. Venderink W, de Rooij M, Sedelaar JPM, Huisman HJ, 
Fütterer JJ. Elastic Versus Rigid Image Registration in Magnetic Resonance Imaging-transrectal Ultrasound Fusion Prostate Biopsy: A Systematic Review and Meta-analysis. Eur Urol Focus. 2018;4:219-227.

61. Halstuch D, Baniel J, Lifshitz D, Sela S, Ber Y, Margel D. Assessment of Needle Tip Deflection During Transrectal Guided Prostate Biopsy - Implications for Targeted Biopsies. J Endourol. 2018;32:252-256.

62. Radtke JP, Schwab C, Wolf MB, Freitag MT, Alt CD, Kesch $\mathrm{C}$, et al. Multiparametric Magnetic Resonance Imaging (MRI) and MRI-Transrectal Ultrasound Fusion Biopsy for Index Tumor Detection: Correlation with Radical Prostatectomy Specimen. Eur Urol. 2016;70:846-853.

63. Villers A, Puech P, Mouton D, Leroy X, Ballereau C, Lemaitre L. Dynamic Contrast Enhanced, Pelvic Phased Array Magnetic Resonance Imaging of Localized Prostate Cancer for Predicting Tumor Volume: Correlation With Radical Prostatectomy Findings. J Urol. 2006;176:24322437.

64. Schreiber D, Wong AT, Rineer J, Weedon J, Schwartz D. Prostate biopsy concordance in a large populationbased sample: A Surveillance, epidemiology and end results study. J Clin Pathol. 2015;68:453-457.

65. Epstein JI, Feng Z, Trock BJ, Pierorazio PM. Upgrading and downgrading of prostate cancer from biopsy to radical prostatectomy: Incidence and predictive factors using the modified gleason grading system and factoring in tertiary grades. Eur Urol. 2012;61:1019-1024.

66. Porpiglia F, DE Luca S, Passera R, Manfredi M, Mele F, Bollito E, et al. Multiparametric-Magnetic Resonance/ Ultrasound Fusion Targeted Prostate Biopsy Improves Agreement Between Biopsy and Radical Prostatectomy Gleason Score. Anticancer Res. 2016;36:4833-4839.

67. van den Bergh RC, Ahmed HU, Bangma $\mathrm{CH}$, Cooperberg MR, Villers A, Parker CC. Novel tools to improve patient selection and monitoring on active surveillance for lowrisk prostate cancer: A systematic review. Eur Urol. 2014;65:1023-1031.

68. Luzzago S, Musi G, Catellani M, Russo A, Di Trapani E, Mistretta FA, et al. Multiparametric Magnetic-Resonance to Confirm Eligibility to an Active Surveillance Program for Low-Risk Prostate Cancer: Intermediate Time Results of a Third Referral High Volume Centre Active Surveillance Protocol. Urol Int. 2018;101:56-64.

69. Ouzzane A, Renard-Penna R, Marliere F, Mozer P, Olivier J, Barkatz J, et al. Magnetic Resonance Imaging Targeted Biopsy Improves Selection of Patients Considered for Active Surveillance for Clinically Low Risk Prostate Cancer Based on Systematic Biopsies. J Urol. 2015;194:350-356.

70. Kamrava M, Kishan AU, Margolis DJ, Huang J, Dorey F, Lieu P, et al. Multiparametric magnetic resonance imaging for prostate cancer improves Gleason score assessment in favorable risk prostate cancer. Pract Radiat Oncol. 2015;5:411-416.

71. Pessoa RR, Viana PC, Mattedi RL, Guglielmetti GB, Cordeiro MD, Coelho RF, et al. Value of 3-Tesla multiparametric magnetic resonance imaging and targeted biopsy for improved risk stratification in patients considered for active surveillance. BJU Int. 2017;119:535-542.

72. Tran GN, Leapman MS, Nguyen HG, Cowan JE, Shinohara $\mathrm{K}$, Westphalen AC, et al. Magnetic Resonance ImagingUltrasound Fusion Biopsy During Prostate Cancer Active Surveillance. Eur Urol. 2017;72:275-281.

73. Gelet A, Crouzet S, Rouviere O, Bratan F, Chapelon JY. Focal treatment of prostate cancer using Focal One device: pilot study results. J Ther Ultrasound. 2015;3:O54. Available from: http://jtultrasound.biomedcentral.com/ articles/10.1186/2050-5736-3-S1-O54

74. von Hardenberg J, Westhoff N, Baumunk D, Hausmann D, Martini T, Marx A, et al. Prostate cancer treatment by the latest focal HIFU device with MRI/TRUS-fusion control biopsies: A prospective evaluation. Urol Oncol. 2018;36:401. e1-401.e9.

75. Valerio M, Shah TT, Shah P, Mccartan N, Emberton M, Arya $\mathrm{M}$, et al. Magnetic resonance imaging-transrectal ultrasound fusion focal cryotherapy of the prostate: A prospective development study. Urol Oncol. 2017;35:150.e1-150.e7.

76. Natarajan S, Jones TA, Priester AM, Geoghegan R, Lieu P, Delfin M, et al. Focal Laser Ablation of Prostate Cancer: Feasibility of Magnetic Resonance Imaging-Ultrasound Fusion for Guidance. J Urol. 2017;198:839-847.

77. Altok M, Kim B, Patel BB, Shih YC, Ward JF, McRae SE, et al. Cost and efficacy comparison of five prostate biopsy modalities: a platform for integrating cost into novelplatform comparative research. Prostate Cancer Prostatic Dis. 2018;21:524-532.

78. Ahmed HU, El-Shater Bosaily A, Brown LC, Gabe R, Kaplan R, Parmar MK, et al. Diagnostic accuracy of multi-parametric MRI and TRUS biopsy in prostate cancer (PROMIS): a paired validating confirmatory study. Lancet. 2017;389:815-822.

79. Venderink W, Govers TM, De Rooij M, Futterer JJ, Sedelaar JPM. Cost-effectiveness comparison of imagingguided prostate biopsy techniques: Systematic transrectal ultrasound, direct in-bore MRI, and image fusion. AJR Am J Roentgenol. 2017;208:1058-1063.

80. Faria R, Soares MO, Spackman E, Ahmed HU, Brown LC, Kaplan R, et al. Optimising the Diagnosis of Prostate Cancer in the Era of Multiparametric Magnetic Resonance Imaging: A Cost-effectiveness Analysis Based on the Prostate MR Imaging Study (PROMIS). Eur Urol. 2018;73:23-30.

81. Westhoff N, Siegel F, Peter C, Hetjens S, Porubsky S, Martini $\mathrm{T}$, et al. Defining the target prior to prostate fusion biopsy: the effect of MRI reporting on cancer detection. World J Urol. 2019;37:327-335.

82. Wang NN, Fan RE, Ghanouni P, Sonn GA. Teaching Urologists "How to Read Multi-Parametric Prostate MRIs Using PIRADSv2": Results of an iBook Pilot Study. Urology. 2019;131:40-45.

83. Kumar V, Bora GS, Kumar R, Jagannathan NR. Multiparametric (mp) MRI of prostate cancer. Prog Nucl Magn Reson Spectrosc. 2018;105:23-40. 